\title{
Evaluating the effectiveness of PE curriculum based on the classroom teaching \\ Feng Chao
}

PE school of Mianyang Normal University, Mianyang, 621000, China

email: 2796479@qq.com

Keywords: Classroom Teaching; Physical Education Course; Effectiveness Evaluation

\begin{abstract}
Physical Education under the new curriculum reform is the development, succession, should draw the essence from traditional education wisdom, promote the perfection of sports teaching practice constantly. In the face of different aspects of the problem and the teaching reform of the "plateau phenomenon", physical education teachers should change ideas, establish confidence, teaching creative, interesting, promote sports curriculum gradually toward the curriculum goal, in order to facilitate the teachers and students together into long, promote the effectiveness of physical classroom teaching improvement.
\end{abstract}

\section{Introduction}

The sports teaching effectiveness specifically refers to teacher follows the teaching of the law, effectively achieve the expected teaching effect, success excited, cause, promote and maintain the students in physical education teaching. In recent years, along with the teaching reform of physical education in our country unceasingly thorough, the concept of effective teaching of PE teaching to a great extent, and gradually gained the approval and attention more and more. Compared with the traditional teaching mode of physical education, effective teaching mode more emphasis on the cultivation of students' interest more can reflect the value of the individual. However, in the physical classroom teaching, effective teaching seriously insufficient, physical education has many problems, which restrict the junior middle school sports teaching efficiency. According to the current situation of physical education teaching in China, in-depth study and improve the effectiveness of classroom teaching and evaluation of physical problems, hope to be able to bring positive effect on physical education.

\section{The Factors of Affecting the Effectiveness of PE Curriculum}

\section{1 students}

\subsection{1 lack of correct understanding for PE Curriculum}

As everyone knows, in the school teaching, sports curriculum is one of indispensable organic component, is an important way to cultivate all-round talents and quality education. However, in the current physical education curriculum teaching, most of the students think that the sports curriculum is a small subject, is not important to the future of education and learning, so perfunctory cope with, participation in physical training and physical exercise of initiative, enthusiasm is not high.

\subsection{2 academic stress}

It is because the students take the great pressure of study, learning very nervous, the mere existence of the major tasks in the heads of some students, but do not know the sports curriculum to the development of physical and mental health play an important role can not be ignored. The heavy academic pressure has great influence on the deviation of understanding of physical education curriculum, so that students lack of interest in sports course is inevitable.

\section{2 family}

Students in the growing process, often have different hobbies, to a certain extent some interest for students to plan for the future. At present, widespread parents look into female bad phenomenon Feng, pushy, most parents only concern is the children's culture course grades, such practices and 
ideas, not only will influence not good to students' values, but also make students love interest was hit, the development of students personality the opportunity was inhibited.

\section{3 teachers}

2.3.1 teaching goal setting lack of scientific

Many physical education teachers, serious lack of teaching objectives of physical education curriculum value, set the goal of classroom teaching has strong randomness, lack of planning in the process of teaching, teaching contents appear randomly change the poor condition of regular, bring the negative influence to the validity of PE teaching.

\subsubsection{Lag of teaching mode}

Under the traditional teaching mode of physical education, PE teachers tend to PE teaching emphasis placed on teaching sports skills and explain the sports knowledge, what I say goes. The phenomenon is common for teachers, the subject position of students has been seriously neglected. In recent years, along with the "value", "attitude", "emotion" began gradually become the evaluation of physical education curriculum teaching standard, physical education teachers to the students' interest in learning seriously, however, in the actual execution, many sports teachers due to the deviation of understanding, define the sports classroom was opened for students interest in learning activities, this makes the "sheep" type of teaching situation.

2.3.3 setup project a single, teaching content boring

In the course of sports teaching, it is because of school facilities and conditions of teachers and professional focus more limited, selective open teaching content and teaching project is single. The vast majority are opened to sport courses exam project Uighur, such as hollow spheres, standing long jump and skip, seldom involving the volleyball, football, basketball and other sports teaching, long time for these boring sports project to be recycled, greatly reduced the physical education for students of learning interest and the participation enthusiasm, make "with heart against adverse conditions".

\section{4 schools}

2.4.1 sports teaching system is not perfect

Many of the extracurricular activities, big break activities and sports teachers in class management system lack of perfection, this makes the part of students and teachers are not serious attitude towards extracurricular activities, activity time, students participate in sports activities of the initiative and mood is not high, it can be said that this is a recessive to the effective sports teaching blow.

\subsection{2 the lack of sports equipment}

Different schools have different equipment, some school facilities facilities relatively complete, in outdoor stadiums and indoor venues are equipped with the appropriate equipment, and has a relatively complete equipment types, but there are still many school ground equipment Co., such as no table tennis room and indoor stadiums, basketball field not too small, track and field, whenever rainy weather had to be indoors during the.

\section{Evaluation of Sports Teaching Effectiveness}

Evaluation of physical education is a specific application of evaluation of teaching in sports discipline, is according to the teaching goal of corresponding standards and sports, the whole process of systematic investigation of physical education, and the advantages and disadvantages and value evaluation, in order to obtain a process improvement and perfection. Can say, the sports teaching evaluation is a combination of flexibility and binding body, sports practice course with nature to technology oriented, taking into account the humanities, Sciences and randomness.

In the current curriculum standard, teaching content and evaluation of the effectiveness of classroom teaching sports selected to help students social adaptation, physiological and psychological health, and promote the different stages of the students to improve their overall level of health, to make students in different level of learning objectives will be considered; personality differences on students to take into account, the full scope to the students, to students in the physical activity participation can benefit equally. At the same time, physical education teaching evaluation 
of the present more attention is paid to the "student oriented", through the teacher's creative work, to enable students to participate in the sports curriculum's study. So in the choice of evaluation of teaching content, teachers need to be integrated to the evaluation content, the realization of the ultimate goal of a content can reach several evaluation. This can not only avoid the complexity of teaching content, but also in the gradual learning of students. In addition, also can be used for non teaching content, as a supplementary evaluation, such as the autonomy of some students, encourage students to evaluate the teaching content of interest.

\section{The Method of Evaluating PE Teaching}

\subsection{The movement of interest group participation}

In the extracurricular activities, teachers can have its own in accordance with the skills and the school hardware facilities, to the sports interest group diversified to offer, in order to developing students' specialty platform are provided, which can effectively reflect the students' interest of sports special length and movement, that the growth and development of students, more it is important to be able to more information rich and accurate to express individual student achievement.

\section{2 sports participation out of school}

In addition to the sports teaching material of the school itself, social participation in physical activity is particularly important as an extracurricular sports activities. Students of the one or two social sports activities to master, at the same time it is a common method in physical exercise, as a content evaluation of physical education, it will arouse the enthusiasm of students to participate in sports activities, the initiative, make teaching evaluation mechanism more perfect.

4.3 the daily records at extracurricular activities

Sports daily extracurricular activity records, such as big break activities, comprehensive activities, sports activities, class-break setting-up exercise and exercise in the morning. Teaching evaluation is not discontinuous, also is not a one-off, but a process of students' development in body and tracking for a considerable period. Active record is for card type descriptions of students in extra-curricular activities and behavior, especially the comprehensive exploration activities and extra-curricular activities, the establishment of specific activities files, clear the students interest hobby, as the evaluation of the effectiveness of teaching physical education curriculum index. The specific method of operation is the establishment of the activity profile for each student, the student to group by group leader, daily record, while teachers need regular conversation with the students, so that it can truly understand the teacher evaluation in extracurricular activities.

\section{The Strategies to Enhance the Effectiveness of Classroom Teaching}

From the general point of view, in the physical classroom teaching deficiencies still exist a series of, there are many that need to be solved as soon as possible, the existence of these problems greatly restrict the effectiveness of physical classroom teaching improvement. Therefore, teachers should explore the reform and the effective measures to strengthen the rise of PE classroom teaching, in order to improve the effectiveness of physical classroom teaching. The following put forward to enhance the effectiveness of physical classroom teaching strategy:

5.1 to stimulate learning interest in sports

In the past the traditional teaching mode of physical education, sports teachers just to take on the teaching mode of teaching, with emphasis on the mastery of sports skills, and which contains to the movement itself fun caused serious neglect, almost no time and space interaction between students and teachers. In order to improve the effectiveness of physical classroom teaching, so teachers should actively and students to engage in sports classroom activities, create a good atmosphere of physical exercise, sports activities will be found in the problem, the right to guide students to solve problems timely, and share the success and failure in sports with the students, attach great importance to cultivating the students sports spirit and personality. In the sports teaching process, teachers should try to give students, mainly in the teaching and curriculum two aspects reflected. 


\section{2 open curriculum resources screening}

In the physical classroom teaching has many open curriculum resources, but all contribute to the implementation of physical education curriculum resources, contribute to the realization of curriculum resources, are the effective resources of physical education teaching. These resources were basically the original form, PE teachers need careful screening, treatment of science, especially to carefully choose the help students life-long sports development, training of the sports curriculum resources. Its main purpose is to exercise the required technical skills, the future citizen sports knowledge reserve, in order to maximize the deepen students' experience and understanding of the physical education curriculum in the learning process.

5.3 strengthen the harmony of multidisciplinary integration

The "harmony" idea of new curriculum standard implementation, can contribute to the development and reform of physical education curriculum. In the new teaching materials of sports, physical education teachers should demand and subject integration times closely combine, to grasp of the longitudinal subject system logic at the same time, the permeability and related sports science and other related disciplines to strengthen. Physical education textbook new focus is the integration of vision and understanding of sports to solve problems, to sports discipline has the characteristics to be outstanding, organic to health, sports participation, social adaptation and mental health goals together, in order to promote students to learn science and systems thinking to solve physical problems, promote comprehensive the physical education reform, the rich humanities accomplishment. Therefore, teachers should be in the new curriculum under the guidance of, pay attention to mutual penetration, sports teaching aspect, attach importance to the harmonious integration of multi discipline. Furthermore, teachers should pay attention to individual differences of students, changes of teaching evaluation mechanism, to strengthen the persistent interest in learning.

\section{Conclusion}

Effective teaching and curriculum reform is not to in some teaching methods to replace the traditional way of teaching, physical education culture under the new curriculum reform is the development, should draw the essence from traditional education wisdom. Needs to be pointed out is, not the "best" way of teaching, in teaching attempt to use and understanding of some kind of teaching approach is wrong, so, in the face of different aspects of the problem and the teaching reform of the "plateau phenomenon", physical education teachers should change ideas, establish confidence, teaching creative, fun promote the sports curriculum, gradually toward the curriculum goal, to grow together to facilitate the realization of teachers and students, promote the effectiveness of physical classroom teaching improvement.

\section{References}

[1] Li Lin. Effective classroom teaching physical education evaluation [J]. China Education Innovation Herald, 2011(33):101-103.

[2] Ji Yuanbo. Expo physical education class teaching efficiency [J]. sports on the background of new curriculum, 2011(13): 38-43.

[3] Zhang Lining. Pay attention to sports teaching building "effective teaching" [J]. modern reading (EDUCATION EDITION), 2011(10): 40-42.

[4] Liu Junxia, Chen Yuxia [J]. new curriculum effective teaching of (on), 2011(6) :46-48.

[5] Chen Kun. How to carry out efficient sports teaching [J]. the friends of students (junior middle school), 2011(6): 55-56.

[6] Shang Wenjin. Constructing effective classroom teaching of middle school sports thinking about the [J]. sport, 2011(7): 86-87. 